This is a postprint version of the following published document:

Llorente, A., Serrano, B. \& Baselga, J. (2017). The Effect of Polymer Grafting in the Dispersibility of Alumina/Polysulfone Nanocomposites.

Macromolecular Research, 25 (1), pp. 11-20.

DOI: $10.1007 / \mathrm{s} 13233-016-4150-1$

(C) Springer Verlag, 2017 


\title{
The Effect of Polymer Grafting in the Dispersibility of Alumina/ Polysulfone Nanocomposites
}

\author{
Amaia Llorente, Berna Serrano*, and Juan Baselga \\ Department of Materials Science and Engineering and Chemical Engineering, Universidad Carlos III de Madrid, Avenida \\ de la Universidad 30, 28911 Leganés, Madrid, Spain \\ *Corresponding Author. E-mail: berna@ing.uc3m.es
}

\begin{abstract}
$\gamma$-Alumina nanoparticles have been modified with polysulfone (PSU) chains via 1,3-dipolar cycloaddi-tion reaction between functionalized alumina with vinyl groups and terminal azide polysulfone chains of two differ-ent molecular weights. Homopolymer nanocomposites have been prepared for the first time by extrusion and microinjection. The effectiveness of the grafts on the dispersiblity has been analyzed in terms of the parameters that govern the wettability between grafted and matrix chains: graft density $(\sigma)$, graft molecular weight $(N)$ at constant matrix molecular weight $(P)$.

The dispersion state and interfacial adhesion of PSU grafted-nanoparticles have been evaluated from laser scanning confocal, FESEM and SEM microscopy. Results show that the incorporation of the modified g-alumina improves the dispersion state in comparison with bare alumina nanoparticles, reducing the aver-age particle size from $5 \pm 9$ to $1.3 \pm 1$ microns. Although aggregates are still present the size of the aggregates are also substantially reduced even with low or moderate graft density used in this work, but further improves the interfacial adhesion between nanoparticle and matrix when long PSU chains are grafted even with low-moderate grafting den-sity. These results can be explained by enthalpic compatibility between polysulfone grafted layer and host polysul-fone matrix
\end{abstract}

Keywords

polysulfone, grafted nanoparticle, dispersion state, nanocomposite.

\section{$\underline{\text { Introduction }}$}

Polysulfone is a high performance engineering polymer, and thanks to its specific properties such as its biocompatibility, transparency, high thermal and chemical stability, mechanical strength, resistance to extreme $\mathrm{pH}$ values and low creep, it is considered one of the most valuable polymers in engineering application, widely used as functional material in biochemical, and medical fields as well as in fuel membranes. ${ }^{1-4}$

However, in some cases, polysulfones and their derivatives can show relatively poor mechanical and wear resistance, so different types of inorganic nanofillers have been incorporated into this polymer, ${ }^{5-8}$ with the aim of expanding its applications through the enhancement of mechanical strength ${ }^{9,10}$ without the loss of desirable physical properties. ${ }^{11-13}$ These characteristics have demonstrated to be very strongly dependent on the dispersion state of the nanoparticles in the polymer matrix. ${ }^{14,15}$ Nowadays it is well established that the dispersion state of nanoparticles, within a polymer matrix, controls the physical and mechanical properties of the nanocomposite. ${ }^{16}$ Thus, beneficial effects on mechanical and physical properties would be improved when stable dispersion of nanoparticles (NPs) is achieved without formation of the aggregates.

The addition of inorganic nanoparticles in a polymer matrix including $\mathrm{Al}_{2} \mathrm{O}_{3}, \mathrm{SiO}_{2}, \mathrm{TiO}_{2}$, etc., has attracted particular interest in the development of advanced polymeric materials. ${ }^{17-19}$ However, the chemical dissimilarity between the inorganic nanoparticle and polymer matrix makes it difficult to disperse the nanoparticles homogeneously. This difficulty may be overcome by surface modification of nanoparticles with organic molecules which screen out particle-particle interactions preventing the formation of aggregates. ${ }^{20,21}$ Surface modification of nanoparticles can be as simple as the addition of short molecules to shield the nanoparticle surface reducing the particleparticle attractions, or through more complex methods based on the grafting of long polymer chains to further optimize the wettability with the matrix. ${ }^{22-24}$ Homopolymer grafted to surface nanoparticles have been widely used to enhance dispersion and mixing into the polymer matrix by creating a chemical compatible layer between the nanoparticle and the polymer matrix ${ }^{25-29}$ Besides, variations in graft density $(\sigma)$ and graft chain length $(N)$ provide different chain conformations of the shell, which affects the dispersion/aggregation of nanoparticles in the matrix. ${ }^{30}$

Sunday et al..$^{31}$ developed a phase diagram to predict the 
stability of polystyrene grafted silica nanoparticles in polystyrene matrices. It is based on the three parameters that govern the dispersion state, $\sigma$ and $P / N$, and shows a complete wetting region with an allophobic dewetting transition at low $\sigma$ and an autophobic dewetting transition at high $\sigma^{32}$ So, a complete wetting region can be reached for moderate $\sigma$ for $P / N \leq 1$. In this work we use the "grafting to" method to graft PSU chains on the nanoparticle surface. This method provides lower graft densities ${ }^{33,34}$ than "grafting from" method, but according to the scaling criterion, an entropic attraction between grafted surfaces exists when $\left(\sqrt{\sigma N}>(N / P)^{2}\right)$ inducing an autophobic dewetting of grafted nanoparticle within polymer melt. ${ }^{35}$ This is because when two grafted nanoparticles with high $\sigma$ come close together the matrix chains are expelled from the interfacial region and consequently the dispersion gets worse. In order to facilitate matrix-grafted chains penetration, either $\sigma$ must be decreased, at the risk of insufficient particle-particle screening or a similar (or lower) molecular weight matrix must be employed $(P / N \leq 1)$. However, a polymer matrix with low molecular weight presents a low technological interest, thus in this work, to obtain a balance between particle-particle attraction and entropic repulsion, an intermediate grafting density has been used to overcome the "autophobic wetting". 31,36

We report on an interesting case where PSU grafted to galumina nanoparticles are immersed in a melted PSU matrix. To our knowledge this is the first time that this system is under study, and it is also the first time a successful route for grafting PSU chains on alumina nanoparticles is reported. Previous attempts to improve the mechanical behavior of PSU were based on the chemisorption of some fatty acids over the surface of $\gamma$-alumina. ${ }^{5}$ It was found that the acids formed nanosized self-assembled structures which were the main reason for the improvement of mechanical properties of PSU, despite of absence of favorable nanoparticle-matrix interaction. In the present work we introduce two beneficial effects to the dispersion state: i) a favorable enthalpic interaction with a favorable thermodynamic (Flory-Huggins) interaction parameter, $\chi{ }^{35}$ because segments of both components, matrix and polymer grafts, do no present disparity; and ii) polymer grafted-nanoparticle introduces both steric repulsive forces to keep the nanoparticle separated and strong adhesion between nanoparticles and polymer matrix by entanglements between the chains of either. ${ }^{37}$

The surface modification process starts with the preparation of vinyl functionalized alumina nanoparticles; subsequently, azide terminated PSU chains (PSU- $\mathrm{N}_{3}$ ) are attached covalently through a "click chemistry" reaction. Two PSU graftedalumina nanoparticles have been prepared: i) a low molecular weight PSU synthesized in this work (short chains) and ii) a commercial PSU with high molecular weight (long chains). Each type of PSU-grafted nanoparticle represents a different state of the phase diagram: short chains: $P / N \gg>1$, and long chains: $P / N \leq 1$. A commercial PSU of high molecular weight was selected as the matrix. Concerning the inorganic nanoparticle, -alumina was selected for both its known biocompatibility and its high strength. ${ }^{38,39}$ The grafted nanoparticles suspension stability was investigated by DLS on nanoparticles without polymer matrix in a good solvent. Microscopy techniques such as Confocal Laser Scanning Microscope (CLSM) and Field Emission Scanning Electron Microscope (FE-SEM) were used to study the dispersion state of various loading of grafted nanoparticle within the PSU matrix. SEM microscopy was used to analyze the interfacial adhesion; it was also evaluated through glass transition $\left(T_{g}\right)$ measurements. It was found that the nanocomposites achieve welldispersed states and are more homogenous than bare alumina nanoparticle. These observations suggest that the incorporation of PSU-grafted $\gamma$-alumina nanoparticles at low loading should be a good way to improve the mechanical behavior of alumina/polysulfone nanocomposites.

\section{Experimental}

Materials. $\gamma$-Alumina nanoparticles were purchased from Sigma-Aldrich with a density of $3.29 \mathrm{~g} \mathrm{~cm}^{-3}$ measured by Helium picnometer, and specific surface area of $180 \mathrm{~m}^{2} \mathrm{~g}^{-1}$ determined by $\mathrm{N}_{2}$ isothermal adsorption and desorption and analyzed by BET method (Micromeritics Gemini VII). The nanoparticles size was also estimated by X-ray diffraction (Supporting Information, Figure S1) with a X'Pert diffractometer $\left(2 \theta\right.$ range from $15^{\circ}$ to $\left.90^{\circ}\right)$ from the broadening of one of the diffraction peak using the Scherrer's formula, showing a mean size of $7.47 \pm 0.07 \mathrm{~nm}$. A commercial PSU (SigmaAldrich) with $M_{n}=16,000 \mathrm{~g} \mathrm{~mol}^{-1}$ and $M_{w}=35,000 \mathrm{~g} \mathrm{~mol}^{-1}$ was used as matrix (named PSU16k). Two PSU with low and high molecular weight (short and long chains respectively) were used as grafting polymers as described below. A schematic representation of the whole procedure is shown in Figure 1. Experimental conditions for each step are described in the following paragraphs.

Vinyl Functionalized Alumina. In a first step the nanoparticles were pre-functionalized with vinyltrimethoxysilane (VTMS) (supplied by Sigma-Aldrich) in order to incorporate double bonds to the surface. The surface functionalization of $\gamma$-alu-

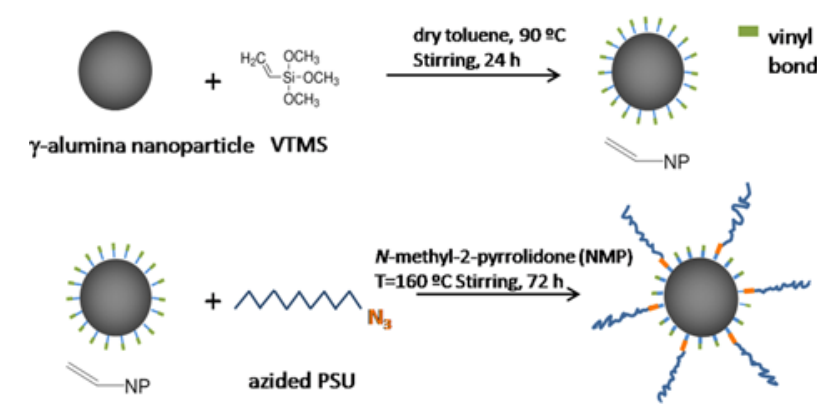

Figure 1. Schematic representation of the synthesis procedure for the preparation of PSU-grafted $\gamma$-alumina nanoparticle. 
mina nanoparticles with VTMS was performed following the protocol established by Gupta for silanes. ${ }^{29}$ Prior any treatment, $\gamma$-alumina nanoparticles were dried at $150^{\circ} \mathrm{C}$ in a vacuum oven for $2 \mathrm{~h}$ to remove adsorbed moisture from the surface. The silanization was performed by adding $6 \mathrm{ml}$ of VTMS to the alumina suspension in dry toluene $(1 \mathrm{wt} \%)$. The reaction mixture was held at $90{ }^{\circ} \mathrm{C}$ for $24 \mathrm{~h}$ under $\mathrm{N}_{2}$ atmosphere and then cooled to room temperature. The product was isolated by centrifugation for $5 \mathrm{~min}$ at 3,000 rpm and washed multiple times via a dispersion-centrifugation process in toluene, until no change in the weight loss (TGA) was observed to ensure no unreacted silane remained. Finally after multiple washings, supernatant was checked by FTIR and no characteristic signal of alkene group from unreacted VTSM was detected. The prepared vinyl-functionalized alumina was dried at $100{ }^{\circ} \mathrm{C}$ in a vacuum oven for $3 \mathrm{~h}$ before analysis.

Synthesis of PSU Short Chains. PSU of low molecular weight was synthesized according to the simple reaction reported by Dizman..$^{40}$ Briefly, bisphenol A (20.0 g, $\left.87.6 \mathrm{mmol}\right)$, bis(p-chlorophenyl) sulfone $(21.0 \mathrm{~g}, 73.1 \mathrm{mmol})$ and dried potassium carbonate, $(12.7,91.9 \mathrm{mmol} \mathrm{g})$ (all purchased from Sigma-Aldrich) in appropriate ratios were added to $200 \mathrm{~mL}$ dimethyl acetamide $/ 25 \mathrm{~mL}$ toluene mixture in a two necked round bottom flask equipped with stir bar and fitted with a condenser and a Dean and Stark trap. The reaction mixture was stirred for $6 \mathrm{~h}$. at reflux under nitrogen atmosphere. Afterwards the reaction was stopped and cooled to room temperature and the solution was filtered to remove the salt. The resultant polymer was precipitated in a mixture of cold methanol/water (4/1). The product was washed several times with water and methanol and dried in a vacuum oven at $100{ }^{\circ} \mathrm{C}$ for about $12 \mathrm{~h}$. A white polymer was obtained (25 g, 15\%). The molecular weight was determined by SEC analysis performed on a Shimadzu LC-20AD pump with an UV-visible detector (Waters 2487 dual $\mathrm{k}$ absorbance detector) and IR detector (Waters 2410 differential refractometer). Tetrahydrofurane (THF) was used as eluent with a flow rate of $1 \mathrm{~mL} \mathrm{~min}^{-1}$ at $35^{\circ} \mathrm{C}$. The molecular weight was calculated with calibration relative to polystyrene standards: $M_{w}=5,633 \mathrm{~g} \mathrm{~mol}^{-1}$ and $M_{n}=2,295 \mathrm{~g} \mathrm{~mol}^{-1}$ with PDI= 2.4. $T_{g}$ by DSC was $126^{\circ} \mathrm{C}$ for synthesized PSU. Those results are in agreement for PSU synthesized by Dizman with similar molecular weight.

Azide Terminated PSU (PSU-N $\mathbf{N}_{3}$ ). The incorporation of azide end groups to both the low and high molecular weight PSU was done using the same chemical approach. It consisted in two steps: first, introduction of the chlorine end group using 2-chloroethyl isocyanate and subsequent transformation of chlorine end group to azide end group using sodium azide. One of the two -OH terminal groups of PSU was intentionally protected by a condensation reaction with 4-chlorophenyl phenylsulfone (purchased from Sigma-Aldrich). The experimental procedure and reaction conditions were similar to that used in the synthesis of PSU, explained before; starting now from $\mathrm{OH}$ terminated PSU and the corresponding mono- chlorine reactive to cap one -OH terminal group of PSU, the reaction was carried out for the two PSU, and the amounts used were: $4 \mathrm{~g}$ of PSU $(0.25 \mathrm{mmol}$ and $1.66 \mathrm{mmol}$ for PSU16k and PSU $2.4 \mathrm{k}$ respectively), the stoichiometric amount of 4-chlorophenyl phenylsulfone for a 1:1 molar ratio $(0.065 \mathrm{~g}$ or $0.42 \mathrm{~g}$, for the reaction with PSU16k and PSU2.4k, respectively), and dried potassium carbonate, (1 g, $7.2 \mathrm{mmol})$ in $100 \mathrm{~mL}$ dimethyl acetamide $/ 25 \mathrm{~mL}$ toluene mixture. The other $\mathrm{OH}$ end group was left unreacted for enabling its chlorination followed by azidation. In a typical reaction, PSU16k (13 g) was dissolved in $100 \mathrm{~mL}$ anhydrous DMF in a Schlenk equipped with a magnetic stir bar at room temperature under nitrogen atmosphere. 2-Chloroethyl isocyanate was next added in excess (2:1) and the mixture was stirred for $24 \mathrm{~h}$. The reaction mixture was poured afterwards into cold methanol to coagulate the product. The product was filtered, washed with additional methanol/water, and dried under vacuum. The success of the reaction was checked by the presence of urethane groups by FTIR for low molecular weight PSU and FTIR-ATR for the higher molecular weight PSU.

Azidation of PSU was done as follows: $10 \mathrm{~g}$ of chlorine terminated PSU was dissolved in DMF in a round-bottom flask equipped with a magnetic stir and an excess of sodium azide was added in a 1:10 molar ratio of PSU chain $/ \mathrm{NaN}_{3}$ ). The mixture was stirred for $24 \mathrm{~h}$ at $60{ }^{\circ} \mathrm{C}$. Afterwards the reaction mixture was poured into cold methanol/water mixture to coagulate the product. The product was filtered, washed with additional methanol/water, and dried under vacuum. In this step the success of the reactions was confirmed by FTIR.

Grafting PSU onto Alumina by Azide/Alkene Cycloaddition Reaction. PSU chains were grafted onto $\gamma$-alumina nanoparticles by an azide/alkene reaction: 1,3 dipolar cycloaddition of azide terminated PSU (PSU-N ${ }_{3}$ ) to the $\mathrm{C}=\mathrm{C}$ double bond inserted on the surface of the nanoparticle. forming the corresponding triazoline. ${ }^{41}$ The procedure was as follows: $9 \mathrm{~g}$ of double bond-functionalized alumina particles (taking into account a graft density of $0.69 \mathrm{~mole} / \mathrm{nm}^{2}$, it corresponds to $1.86 \mathrm{mmol}$ of vinyl functionalities grafted onto alumina nanoparticles) were well dispersed in $50 \mathrm{~mL}$ of $\mathrm{N}$-methyl-2-pyrrolidone (NMP) (purchased from Sigma-Aldrich). Then a solution of $3.72 \mathrm{mmol}$ of PSU-N $\mathrm{N}_{3}$ in $250 \mathrm{~mL}$ of NMP was added, resulting in a 1:2 vinyl/PSU-N ${ }_{3}$ molar ratio. The reaction mixture was stirred at $160{ }^{\circ} \mathrm{C}$ for $72 \mathrm{~h}$ under nitrogen atmosphere. After reaction time, the slurry mixture was centrifuged and washed several times with NMP to eliminate the unreacted $\mathrm{N}_{3}$-PSU.

Nanocomposite Fabrication. These PSU grafted nanoparticles (denoted as PSU2.4k_ $\mathrm{Al}_{2} \mathrm{O}_{3}$ and PSU16k_ $\mathrm{Al}_{2} \mathrm{O}_{3}$ ) were incorporated into a matrix polymer (PSU16k, $M_{n}=16,000$ gmol $^{-1}$ ) by melt compounding at 2, 5, and $10 \%$ (wt/wt) loadings and a subsequent injection process.

The PSU matrix (PSU16k) and the nanoparticles (bare $\gamma$ alumina or grafted $\gamma$-alumina) were mixed in a Haake Minilab micro compounder with two coextruded rotors at a processing temperature of $360^{\circ} \mathrm{C}$, recirculation time $10 \mathrm{~min}$ and screw 
rotating speed $150 \mathrm{rpm}$. The extruded pellets of neat PSU16k and each nanocomposite set of the different compositions were molded using a Battenfeld Microsystem 50 micro holding machine to obtain miniature dog-bones specimens $(16 \times 3 \times 1)$ and circular specimens $(\mathrm{d}=7 \mathrm{~mm}, \mathrm{t}=0,45 \mathrm{~mm})$. Molding conditions were: mold temperature $135^{\circ} \mathrm{C}$, Injection temperature and pressure $345^{\circ} \mathrm{C}$ and 500 bar respectively, cycle time: $20 \mathrm{~s}$. Before testing all specimens were kept at $150{ }^{\circ} \mathrm{C}$ for $1 \mathrm{~h}$ to remove residual stresses.

The following abbreviations are used in this paper: PSU/ $\mathrm{xAl}_{2} \mathrm{O}_{3}$ where $\mathrm{x}$ can be 2, 5 or 10 , and represent the $\mathrm{wt} \% \gamma$ alumina loading. PSU/PSU2.4k_ $\mathrm{xAl}_{2} \mathrm{O}_{3}$ and PSU/PSU16k_x$\mathrm{Al}_{2} \mathrm{O}_{3}$ for grafted alumina systems for the nanocomposites containing PSU2.4k grafted (short chains) and PSU16k grafted (long chains) $\gamma$-alumina nano reinforcement, at the same amount (with $\mathrm{x}=2,5$, or 10 ).

Characterization. The as-received g-alumina (bare alumina) and modified nanoparticles (VTMS-alumina and PSU grafted alumina) were characterized by thermogravimetric analysis (TGA) using a Perkin Elmer STA 6000: heating scans at $10^{\circ} \mathrm{C}$ $\mathrm{min}^{-1}$ from $50^{\circ} \mathrm{C}$ to $900^{\circ} \mathrm{C}$ in a flow of air $\left(40 \mathrm{~mL} \mathrm{~min}^{-1}\right)$. Before measurements, nanoparticles were dried in vacuum oven at $150^{\circ} \mathrm{C}$ for $12 \mathrm{~h}$. Fourier transform infrared spectroscopy (FTIR) of modified alumina were performed using a Perkin Elmer Spectrum GX-2000 spectrometer with a resolution of $1 \mathrm{~cm}^{-1}$ in a scale range of $4000-400 \mathrm{~cm}^{-1}$. The bare g-alumina and modified alumina samples were diluted in dried $\mathrm{KBr}$ and pressed in pellet. FT-Raman measurements were also performed for PSU grafted nanoparticles using a Bruker (D) FRA-106/S component attached to an EQUINOX 55 spectrometer. Spectra shown here are an average of 100 scans at $4 \mathrm{~cm}^{-1}$ resolution.

Cryogenic fracture surfaces were inspected by SEM (Phillips XL30 scanning electron microscope) to observe the final microstructure in the nanocomposite. Samples were prepared by submerging them in liquid $\mathrm{N}_{2}$, breaking it, and sputter-coating it with gold before analysis to prevent charging.

Confocal Laser Scanning Microscope (CLSM) observation of the nanocomposite was made on an inverted microscopy (LSM 5 PASCAL, Carl Zeiss, Germany) with a $458 \mathrm{~nm}$ Ar laser in reflection mode. Observation was made on injection molded circular specimens $(\mathrm{d}=7 \mathrm{~mm}, \mathrm{t}=0.45 \mathrm{~mm})$. FESEM microscopy (FEI Nova NANOSEM 230) was also used to observe the samples investigated by CLSM. Glass transition temperatures $\left(T_{g}\right)$ of PSU and its nanocomposites were determinated by DSC (Mettler Toledo 822): 5-10 mg of the samples were hermetically sealed in aluminum pans and measured in the range $25-225^{\circ} \mathrm{C}$ at $10^{\circ} \mathrm{C} \mathrm{min}^{-1}$ under $10 \mathrm{~mL} \mathrm{~min}^{-1} \mathrm{~N}_{2} ; T_{g}$ values were obtained from the inflection points of the DSC curves in the second heating scan after erasing the thermal history.

\section{Results and Discussion}

Synthesis of Grafted Nanoparticles.

Vinyl Functionalized Alumina: FTIR and Raman spec-

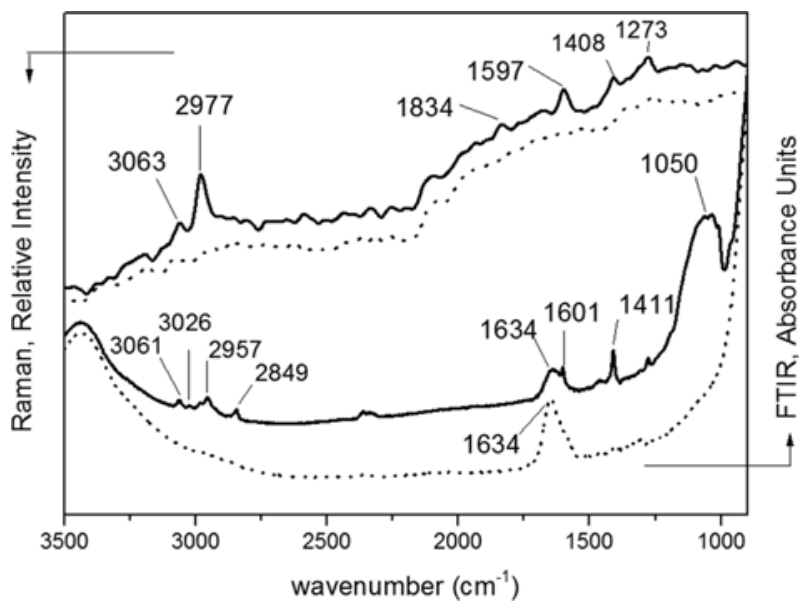

Figure 2. FTIR and FT-Raman spectra of the bare alumina (dotted line) and the vinyl functionalized nanoparticles (solid line).

troscopy were used to confirm the presence of the double bond group on alumina surface. The FTIR spectra of both bare and modified g-alumina are shown in Figure 2. The broad peak above $3200 \mathrm{~cm}^{-1}$ corresponds to the stretching mode of $\mathrm{OH}$ bonded to $\mathrm{Al}(\mathrm{Al}-\mathrm{OH})$. The frequency at $1050 \mathrm{~cm}^{-1}$ is related to the symmetrical bending vibrations of hydrogen bands, and below $1000 \mathrm{~cm}^{-1}$ corresponds to Al-O vibration modes. The peak at $1634 \mathrm{~cm}^{-1}$ is due to the -OH bending vibration of adsorbed water. All of these are characteristic peaks of alumina widely reported in the literature. ${ }^{42,43}$ The dried vinyl functionalized nanoparticles show absorbance bands of both, bare alumina and VTMS. The peaks at $3026 \mathrm{~cm}^{-1}$ and $3061 \mathrm{~cm}^{-1}$ are assigned to $\mathrm{C}-\mathrm{H}$ stretching and $\mathrm{CH}_{2}$ asymmetric stretching for the $\mathrm{CH}=\mathrm{CH}_{2}$ group, respectively. ${ }^{29,44}$ The peak at $2849 \mathrm{~cm}^{-1}$ has been also assigned to stretching vibrations of the vinyl group. ${ }^{44}$ The peak observed at $1601 \mathrm{~cm}^{-1}$ is characteristic of vinyl vibrations for the $\mathrm{Si}-\mathrm{CH}=\mathrm{CH}_{2}$ group. Finally the appearance of a peak at $1411 \mathrm{~cm}^{-1}$, assigned to the in-plane deformation of $=\mathrm{CH}_{2}$ confirms the presence of VTMS moiety. In this work, we will focus our attention on characteristic bands of vinyl groups such as $\mathrm{C}=\mathrm{C}$ stretching vibrations at $1601 \mathrm{~cm}^{-1}$ and the peak at $1411 \mathrm{~cm}^{-1}$ which provide us sufficient information about this functional group.

The Raman (also shown in Figure 2) spectrum, also supports the FTIR results, as the peak observed at $3063 \mathrm{~cm}^{-1}$ is characteristic of the $\mathrm{CH}_{2}$ asymmetric stretching for the $\mathrm{CH}=\mathrm{CH}_{2}$ group. Also we can observe peaks reported for VTMS such as $2977,1597,1408$, and $1273 \mathrm{~cm}^{-1}$ assigned to symmetric stretching $\left(=\mathrm{CH}_{2}\right)$, stretching $(\mathrm{C}=\mathrm{C})$ in-plane deformation $\left(=\mathrm{CH}_{2}\right)$ and in plane deformation $(=\mathrm{CH})$ respectively. ${ }^{45}$

Synthesis of Azide-Terminated Polysulfone (PSU-N ${ }_{3}$ ). The transformation of the -OH end-group of PSU into azide group was evaluated by FTIR. The first step for the azidation process involves the chloration of the $\mathrm{OH}$ terminal group of PSU chains. 2-Chloroethyl isocyanate was used for this purpose. The isocyanate groups can easily react with -OH con- 
taining compounds as it was demonstrated by $\mathrm{Li}^{42}$ in the modification of alumina nanoparticles with 4,4'-methylenebis(phenyl isocyanate) (MDI), even its ability to react with phenolic groups is also reported. ${ }^{46}$ After that, the terminal chlorine group was replaced by an azide group through nucleophilic substitution. FTIR spectrum was used to confirm this reaction, showing the changes in the absorbing frequencies of the PSU and PSU-N ${ }_{3}$. The successful attachment of azide terminal group was evidenced by the presence of the characteristic peak of $\mathrm{C}=\mathrm{O}$ from urethane group and $\mathrm{N}=\mathrm{N}$ from azide group. Both characteristic signals appear in the spectrum at very low intensity, and although FTIR was chosen because it is a highly sensitive technique which is able to examine molecules that are attached at very low concentrations, the characteristics peaks of the product of the reaction were of a too small intensity to carry out a quantitative analysis. The peak at $2120 \mathrm{~cm}^{-1}$ assigned to $\mathrm{N}=\mathrm{N}$ asymmetric stretching, ${ }^{47}$ as well as the vibrational bands at $1675 \mathrm{~cm}^{-1}$ assigned to the $\mathrm{C}=\mathrm{O}$ urethane stretching can be used to confirm the terminal azide functionalization. (FTIR included in Figure S2). For higher molecular weight PSU (PUS16k) characteristics signals in FTIR spectra are even less intense (not shown) because of the greater ratio monomer/terminal group.

Grafting PSU on $\boldsymbol{\gamma}$-Alumina Nanoparticles. 1,3 Dipolar cycloaddition azide/alkene type methodology was used to graft PSU on $\gamma$-alumina nanoparticles surface. At this point we must clarify that double bonds like vinyl group are tolerated to a certain extent, given that they are not electronically activated, since there is no substituent on the $\mathrm{C}=\mathrm{C}$ double bond to accelerate the reaction. ${ }^{48}$ It is well known that the reaction with electron-withdrawing substituents on the azide group is also applicable for rate-accelerating azide/alkene type reaction. ${ }^{49}$ Nevertheless, it has been reported that azide groups form triazoline compound reacting with vinyl bond at elevated temperature (higher than $80{ }^{\circ} \mathrm{C}$ ). ${ }^{50}$ Hence, it is possible to obtain adequate yields of triazoline compound if the temperature and time of the reaction are optimized, i.e., at $180{ }^{\circ} \mathrm{C}$ and $72 \mathrm{~h}$. The grafted PSU nanoparticles were characterized by FTIR and TGA for the two polysulfone chains used in this work. The FTIR spectrum of VTMS-alumina and PSU grafted alumina are presented in Figure S3 of SI, where it can be clearly observed that the characteristic bands do not appear in bare alumina. We confirmed the grafting of the PSU chains by the decrease of the peaks at $1410 \mathrm{~cm}^{-1}$ and $1602 \mathrm{~cm}^{-1}$ assigned to $-\mathrm{C}=\mathrm{C}$ - when the grafting reaction takes place.

After grafting process the obtained product contains the triazoline group. ${ }^{48}$ However, in most cases triazolines are unstable and decompose to form an aziridine or mostly an imine structure. This fact can be confirmed by the presence of a new peak about $1652 \mathrm{~cm}^{-1}$ assigned to imine $-\mathrm{C}=\mathrm{N}$ stretching. ${ }^{51}$ However, this band overlaps with bare alumina surface -OH bending at $1634 \mathrm{~cm}^{-1}$ as it can be clearly observed in Figure 3 . To ascertain the presence of imine groups the intensity of the two characteristic $-\mathrm{OH}$ bands at $3450 \mathrm{~cm}^{-1}$ (stretching) and

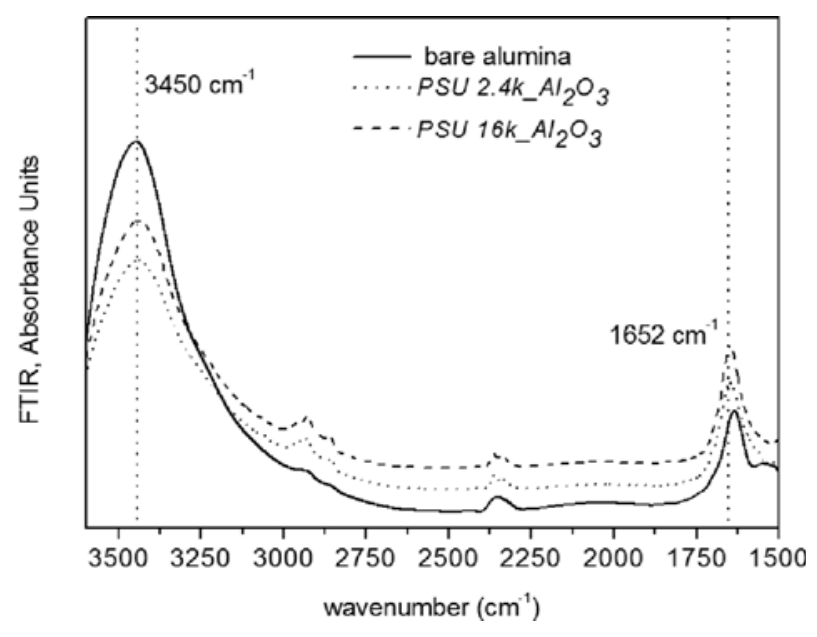

Figure 3. FTIR spectra of bare alumina, PSU2.4k and PSU16k grafted alumina nanoparticle.

$1634 \mathrm{~cm}^{-1}$ (bending) was compared: $I_{1634} / I_{3450}$ was 0.27 for bare alumina while $I_{1652} / I_{3450}$ increased to 0.47 and 0.51 for PSU16k and PSU2.4k respectively. This finding confirms the presence of imine groups on the surface of alumina.

TGA Analysis. Quantification of the vinyl group content on the alumina surface is essential to control the subsequent polymer grafting; this is commonly performed by TGA analysis. However, instead of monolayer, trimethoxy silanes may form multiple layers depending on the reaction conditions; consequently, TGA must be complemented with dynamic light scattering (DLS) measurements in order to obtain the hydrodynamic volume and to estimate the thickness of the layer formed during silanization. TGA curve (see Figure S4 of SI) shows the weight loss of the alumina nanoparticles when heated to $900{ }^{\circ} \mathrm{C}$. The observed weight loss below $100{ }^{\circ} \mathrm{C}$ was attributed to the loss of water. A further weight decrease in modified nanoparticles is related to organic group decom-

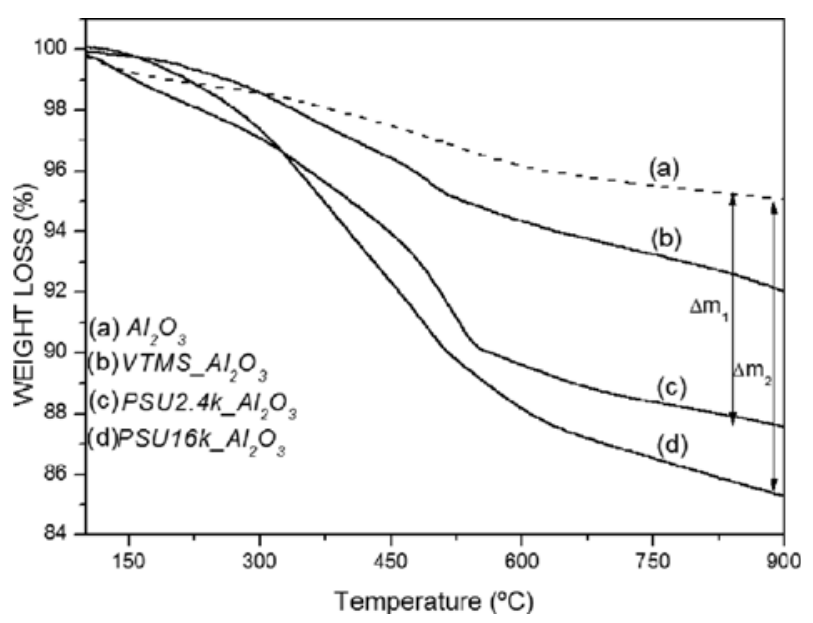

Figure 4. TGA curves for bare alumina and functionalized alumina with VTMS and PSU grafted alumina nanoparticles (flow of air $40 \mathrm{~mL} \mathrm{~min}^{-1}$ ). 
position and $\mathrm{Al}-\mathrm{OH}$ group dehydroxylation. According to TGA experimental data, bare g-alumina particles showed a weight loss of $5 \mathrm{wt} \%$ and VTMS- $\mathrm{Al}_{2} \mathrm{O}_{3}$ a weight loss of $8 \mathrm{wt} \%$. Here, we used the same procedure used by Ash $e t a .^{52}$ to calculate the amount of silane on the surface, and a value of 0.69 molecules per $\mathrm{nm}^{-2}$ was obtained; this value agrees with other reported surface modification processes for silica/trimethoxysilane such as trimethoxy mercaptopropylsilane where it was demonstrated that a trialkoxysilane molecule, on the average, reacts with two $\mathrm{OH}$ groups on the alumina surface. ${ }^{53}$ Assuming a maximum quantity of $4-\mathrm{OH}$ groups $\mathrm{nm}^{-2}$, our result indicates that about $65 \%$ of the surface $-\mathrm{OH}$ groups are reacted with VTMS forming a silane monolayer. ${ }^{54}$

TGA curves for PSU2.4k_ $\mathrm{Al}_{2} \mathrm{O}_{3}$ and PSU16k_ $\mathrm{Al}_{2} \mathrm{O}_{3}$ are presented in Figure 4 and show an increased weight loss due to the decomposition of the grafted PSU chains, indicating successful attachment via alkene-azide cycloaddition in both cases. Calculated graft density using the organic weight loss $\Delta m_{1}$ and $\Delta m_{2}$ (see Figure 4) indicate that not all the vinyl groups were reacted because the graft densities obtained are less than the density obtained for the silane grafting, being 0.069 chains $\mathrm{nm}^{-2}$ and 0.015 chains $\mathrm{nm}^{-2}$ PSU2.4k and PSU16k respectively. These values are in agreement with the reported range for the "grafting to" method where an interval of 0.0010.05 chains $\mathrm{nm}^{-2}$ corresponds to a "moderate density" for which it is possible complete wetting ${ }^{55}$ as it will be discussed below.

Polymer Grafted Configuration. The majority of experimental and theoretical studies on the chain configuration of polymers grafted to surfaces have been carried out with polystyrene ( $\left.\mathrm{PS}, \mathrm{SiO}_{2}\right)$, poly(dimethylsiloxane) and to a lesser extent with polymers such as poly (methyl methacrylate) (PMMA, $\mathrm{Fe}_{3} \mathrm{O}_{4}, \mathrm{TiO}_{2}$ ) or 1,4-polyisoprene (PI, $\left.\mathrm{SiO}_{2}\right){ }^{31,36,56-60}$ The first study utilizing PSU grafted to an inorganic nanoparticle is the present work.

The conformation of polymers end-grafted to the surface of a nanoparticle dramatically changes with graft density and molecular weight. ${ }^{56,58,61}$ At low graft densities the chains are arranged in the shape of a mushroom, and the distance between grafting points is higher than the height reached by the chain (h). On the contrary, high graft density causes chain stretching, so $h$ reaches values greater than the distance between grafting points. "Moderate densities" achieve an intermediate situation. From the graft densities calculated above and assuming Gaussian behavior, a mushroom-like conformation may be realistic for both PSU grafts (short and long chains). Thus, the con- formation that the chain should adopt a priori will be similar to a random coil in a bulk melt with a height twice the radius of gyration ( $h \cong 2 R_{g}$ ) because each chain does not interact with adjacent chains. For a random coil conformation, the radius of gyration is given by $R_{g}=a(N / 6)^{1 / 2}$. ${ }^{62}$ Where $N$ is the number of statistical elements $\left(N=M / m_{n}\right)$, with $m_{n}=111$ for PSU, and $a$ is their length (for PSU it is $0.5 \mathrm{~nm}$ ) ${ }^{63}$ With these data, assuming Gaussian grafts and following the same reasoning as Schulz and Horbach, ${ }^{64}$ calculated $R_{g}$ values are 0.9 and $2.4 \mathrm{~nm}$ for PSU2.4k and PSU16k, respectively.

Due to the low graft density, the volume fraction of polymer chains on the surface is still low; therefore, the elastic free energy is approximately Gaussian. Tchoul ${ }^{65}$ and Henn ${ }^{66}$ reported an expression to calculate the average distance between grafting sites $\left(D_{g s}\right)$ in $\mathrm{nm}$ as a function of graft density $(\sigma), D_{g s}=1 / \sqrt{\sigma}$. With this expression we have calculated a distance of $3.8 \mathrm{~nm}$ and $8.2 \mathrm{~nm}$ for short and long PSU grafts respectively. Table I summarizes calculated parameters used to understand the regime of the PSU grafted chains. In addition to graft density, the height $(h)$ depends on the chain length and the curvature of the nanoparticles as Dukes ${ }^{56}$ reports. In both cases, $R_{g}$ adopts a lower value than the distance between grafting sites $\left(D_{g s}\right)$. This means neighboring grafted chains could not overlap with each other. However it is well known that the structure of polymers end-grafted to a solid surface differs from the corresponding free chains, their conformation changes drastically. ${ }^{62}$ According to results, in either of the two types of grafts the polymer layer is not crowded i.e. the chains are not constrained and are not forced to stretch away from the surface to form a "polymer brush". However, the molecular weight for the large graft (PSU16 $\mathrm{k}_{-} \mathrm{Al}_{2} \mathrm{O}_{3}$ ) is sufficiently large and equal to that of the matrix, and in a good solvent or in the melt the grafted chains should adopt a conformation suitable to entangle with the matrix free chains. Therefore, partial wetting from matrix chains is expected for PSU16k sample. For sample PSU2.4k, it may not be possible to form entanglements with the matrix free chains due to the shorter length of the grafts.

The grafting PSU layer thickness $(h)$ has been also estimated from DLS measurements of the average hydrodynamic diameter $\left(d_{h}\right)$ in NMP solvent for the two functionalized particles and the corresponding size of bare alumina (aqueous dispersion at $\mathrm{pH}=3)$. Layer height was calculated as $h_{D L S}=\left(d_{h}-d_{h, \text { alum }}\right) / 2$, where $d_{h, \text { alum }}$ is the corresponding diameter for pure alumina without functionalization. Results are presented in Table I

Table I. Number of Statistical Segments, $N$, Graft Density, $\sigma$, Distance Between Grafting Sites, $D_{g s}$, Estimated Radius of Gyration, $R_{g}$, Hydrodynamic Diameter, $d_{D L S}$, and Calculated Height from DLS Measurements, $h_{D L S}$, for the Short and Long PSU Grafts

\begin{tabular}{ccccccc}
\hline Sample & $N$ & $\sigma\left(\mathrm{nm}^{-2}\right)$ & $D_{g s}(\mathrm{~nm})$ & $R_{g}(\mathrm{~nm})$ & $d_{D L S}(\mathrm{~nm})(\mathrm{PDI})^{a}$ & $h_{D L S}(\mathrm{~nm})$ \\
\hline $\mathrm{Al}_{2} \mathrm{O}_{3}$ & - & - & - & - & $108(0.3)$ & - \\
PSU2.4k_ $\mathrm{Al}_{2} \mathrm{O}_{3}$ & 21 & 0.069 & 3.8 & 0.9 & $121(0.22)$ & 6.5 \\
PSU16k_ $\mathrm{Al}_{2} \mathrm{O}_{3}$ & 144 & 0.015 & 8.2 & 2.4 & $137(0.28)$ & 14.5 \\
\hline
\end{tabular}

${ }^{a} \mathrm{PDI}=$ Polydispersity index. 
where it can be clearly observed that $h_{D L S}$ is larger than $2 R_{g}$ for the two grafts. It seems therefore that chains are stressed away from the surface and this behavior can be attributed to coil expansion due to the good solvent quality of NMP.

Hasegawa et al. showed experimentally that in an encounter of two proximate particles bearing a graft density above a certain critical value $\left(\sigma_{c}\right)$ interface disappears decreasing the free energy. ${ }^{67}$ As a consequence an attractive force between the particles appears inducing aggregation. Using the same expression that Hasegawa proposed to estimate the critical graft density $\left(\sigma_{c} \cong N^{-1 / 2} \alpha^{-2}\right)$, the obtained values were 0.87 $\mathrm{nm}^{-2}$ and $0.33 \mathrm{~nm}^{-2}$ for PSU2.4k and PSU16k, respectively, well above the data obtained in this work. Therefore, as a first approximation, it seems reasonable to predict an enhancement effect in the dispersion state of the two systems studied here although, in general, the critical graft density will depend on other variables (solvent quality and matrix molecular weight). ${ }^{68}$

Dispersion State of PSU Grafted $\mathrm{Al}_{2} \mathrm{O}_{3}$ in PSU Matrix. Often transparency and mechanical properties of polymer reinforcement by inorganic nanoparticle are determined by the dispersion state. In order to examine the $\gamma$-alumina nanoparticle dispersion within the PSU matrix, morphology and microstructure of the nanocomposites were examined using SEM, FE-SEM microscopy and Confocal Laser Scanning Microscopy (CLSM) in reflection mode. The micrographs were taken from the samples obtained from injection molding of polymer and nanoparticle mixtures.

Fracture surfaces of the PSU and its nanocomposites at $2 \%$ wt loading were examined by SEM and are shown in Figure 5. SEM micrograph of neat PSU (Figure 5(a)) shows a smooth surface characteristic of ductile materials, while

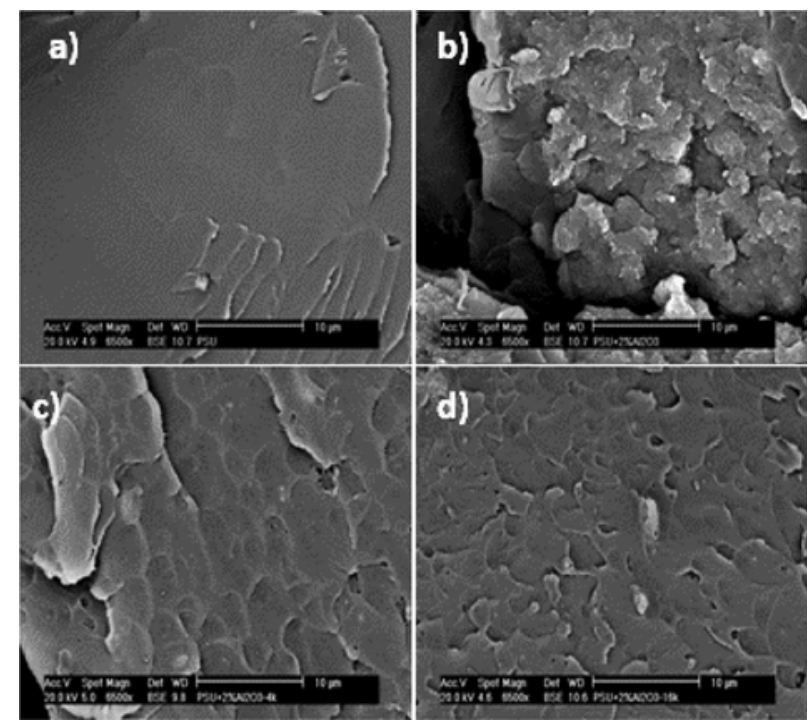

Figure 5. SEM images of the nanocomposite, where (a) shows PSU; (b) shows nanocomposite with bare alumina at 2\%; (c) and (d) show nanocomposites with PSU grafted alumina with PSU2.4k at $2 \mathrm{wt} \%$ and PSU16k at $2 \mathrm{wt} \%$, respectively. by adding bare alumina surface irregularities are observed throughout whole sample, attributed to interface broken during the fracture process (Figure 5(b)). It would be expected since any particle, could act as a discontinuity, hence as a source of stress concentrations during fracture creating a weak and brittle materials. These results demonstrate not only the insufficiently interfacial interaction between the nanoparticles and the PSU matrix, but also the poor toughening capability of the nanocomposite. However, different morphologies were obtained in the case of nanocomposites with PSU grafted alumina (Figure 5(c)-(d)), where particles appear to be uniformly dispersed and surrounded by plastically deformed polymer in a cavity around 1 micron of size, in a similar way that found it in our previous work using fatty modified alumina as nanoreinforcement in a PSU matrix. ${ }^{5}$ However in this case, connectivity with the matrix for both grafted nanoparticle is observed, and even more for the large grafted nanoparticle
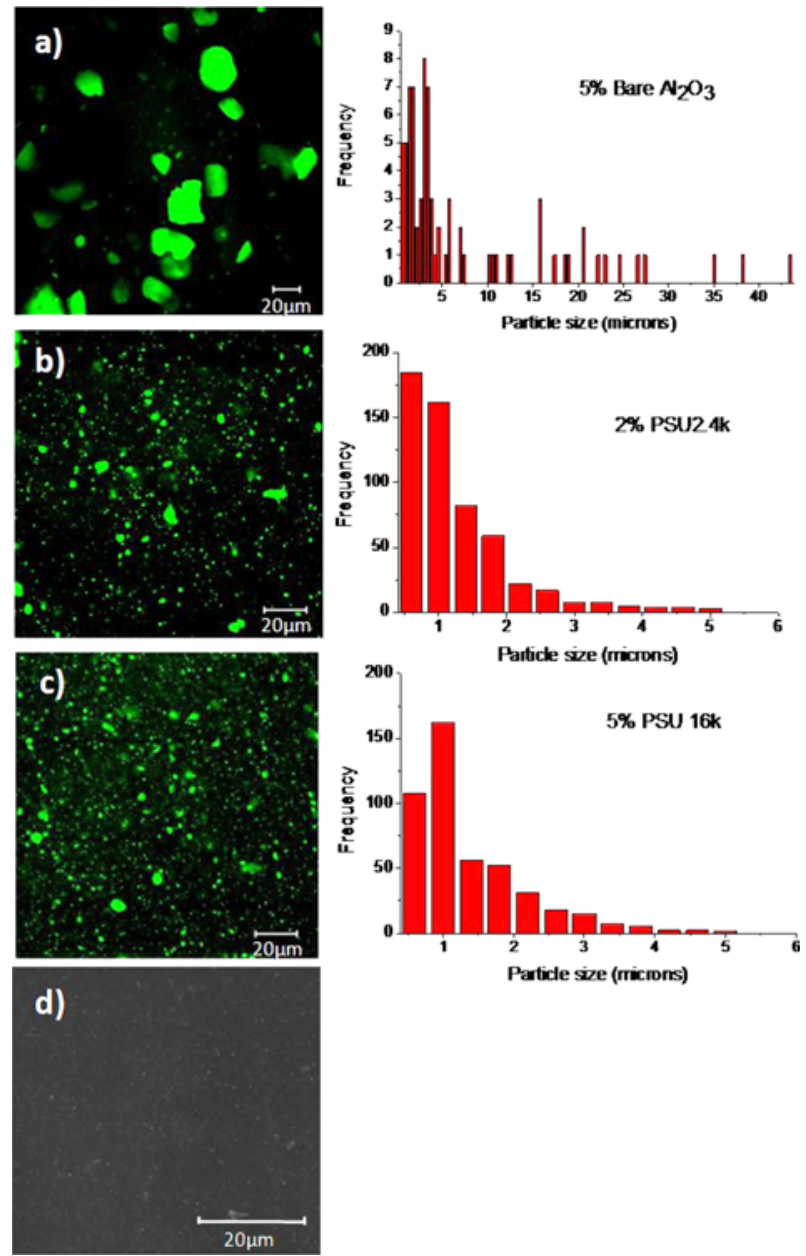

Figure 6. CLSM images of the nanocomposite with black background consisting of PSU matrix and bright areas assigned to alumina aggregates. (a) Nanocomposite with bare alumina at 5\%; (b) nanocomposite with PSU2.4k_ $\mathrm{Al}_{2} \mathrm{O}_{3}$ at $2 \mathrm{wt} \%$; (c) nanocomposite with PSU16k_ $\mathrm{Al}_{2} \mathrm{O}_{3}$ at $5 \mathrm{wt} \%$; (d) FE-SEM image for the same nanocomposite and same surface as the sample used in (c). 
(PSU16k_ $\mathrm{Al}_{2} \mathrm{O}_{3}$ ). An enhancement in the interfacial adhesion it seems achieved during melt mixing and maintained after cooling. These new interactions contribute to the increase of the entanglement density of the matrix and consequently an increasing of ductility of the material is expected.

In order to examine the $\gamma$-alumina nanoparticle dispersion within the PSU matrix the surface of injection molded specimens was examined using FE-SEM microscopy and CLSM. In Figure 6 we present these images for nanocomposites with bare $\mathrm{Al}_{2} \mathrm{O}_{3}$ and with PSU2.4k_ $\mathrm{Al}_{2} \mathrm{O}_{3}$ and PSU16k_ $\mathrm{Al}_{2} \mathrm{O}_{3}$ at 2 and $5 \mathrm{wt} \%$ loading. It can be observed that CLSM images present higher sharpness and contrast than conventional optical images due to the high reflectivity of alumina. The differences between modified and bare alumina are clearly detected. Bare alumina system presents large agglomerates while PSU grafted alumina exhibit a marked improvement on the dispersion state, characterized by a much smaller aggregate size and uniform distribution. Results of image analysis are presented in Table II and Figure 6, in which the distribution of aggregate size is presented for some selected compositions; it must be noticed that particles below the optical resolution limit of the confocal microscope $(290 \mathrm{~nm})$ cannot be detected. It can be clearly observed a reduction in aggregate average size and also in the maximum aggregate size for modified alumina nanocomposites. Numerical data are presented in Table II in which it can be observed an eight fold reduction in agglomerate size. However, no major differences have been found in the dispersion state for each type of grafting irrespective of the loading. A representative FE-SEM image is presented in Figure 6(d) for PSU16k at $5 \mathrm{wt} \%$ loading, where it can be also observed that alumina nanoparticles form agglomerates but they are homogeneously dispersed in the polymer matrix and their sizes are estimated to be about 1 micron. Although there is no evidence of isolated nanoparticles, CLSM and FE-SEM micrographs indicate that the distance between aggregates is much larger than their diameter.

Considering the moderate or low graft density obtained here and the matrix chain length/graft chain length ratio $(P / N)$, our system is close to the boundary allophobic/complete wetting regions as defined in the wetting map for nanoparticle stability in polymer melts predicted by Green et al., Sunday

Table II. Aggregate Average Size for Bare and Modified Alumina Nanocomposites

\begin{tabular}{ccc}
\hline System & $\begin{array}{c}\text { Composition } \\
(\text { wt } \%)\end{array}$ & $\begin{array}{c}\text { Average Size } \\
\text { (microns) }\end{array}$ \\
\hline PSU/2 $\mathrm{Al}_{2} \mathrm{O}_{3}$ & 2 & $5 \pm 9$ \\
PSU/PSU2.4k_2 $2 \mathrm{Al}_{2} \mathrm{O}_{3}$ & 2 & $1.3 \pm 1$ \\
PSU/PSU16k_2 $2 \mathrm{Al}_{2} \mathrm{O}_{3}$ & 2 & $1.3 \pm 0.8$ \\
PSU/5 $\mathrm{Al}_{2} \mathrm{O}_{3}$ & 5 & $7.7 \pm 9$ \\
PSU/PSU2.4k_2 $\mathrm{Al}_{2} \mathrm{O}_{3}$ & 5 & $1.2 \pm 1.3$ \\
PSU/PSU16k_2 $\mathrm{Al}_{2} \mathrm{O}_{3}$ & 5 & 1.30 .8
\end{tabular}

et al. and also used by Akcora et al. ${ }^{24,31,36}$ As indicated by the wetting map the short PSU grafts with a moderate graft density $\left(0.069\right.$ chain $\left.\mathrm{nm}^{-2}\right)$ could be within the well-dispersed region, but the entropic penalty for the matrix to penetrate the brush would make impossible to achieve complete wetting. This usually occurs for moderate or low grafted layers $\left(\sigma N 1 / 2^{1 / 2}<1\right)$ provided that $P$ is large enough. On the contrary, PSU16k $\mathrm{Al}_{2} \mathrm{O}_{3}$ with lower graft density $\left(0.015\right.$ chain $\left.\mathrm{nm}^{-2}\right)$ should be closer to the well-dispersed region because $N=P$. Nevertheless from confocal images the reality is that both PSU2.4k and PSU16k grafted nanoparticles behave in a similar way: micro-sized aggregates are formed in both cases although a clear improvement in the dispersion state is obtained compared to bare alumina.

To reach the same dispersion state for both grafts being so different their $\sigma$ and $N$ values may seem a priori incongruous. However, this behavior is because the dispersion state is governed by a balance between enthalpic attraction between alumina cores and entropic effects related with steric hindrance. Thus, matrix chain partial penetration or wetting takes place for longer PSU grafts even at low grafting density, making it more compatible with the matrix. For shorter grafts, although the length chain is not sufficient, the graft density seems high enough to screen partially the filler core/core van der Waals attractions.

Furthermore, it is also reported that polydispersity of the grafted polymer improves the penetration of matrix chains into the grafted layer of the nanoparticle. ${ }^{69}$ This is because increasing polydispersity relieves the higher monomer crowding in the grafted layer implying higher accessibility by the matrix chains. Both, the PSU matrix and the PSU grafts used here are polydisperse, which helps the stabilization of the nanoparticles in the polymer matrix.

However, the theoretical approach to the stability of grafted nanoparticles in homopolymer matrices has been developed for flexible polymers such as polystyrene or polysiloxane. ${ }^{31,36}$ Although conformational parameters of polysulfone correspond to a flexible polymer (small values of steric hindrance and Flory's characteristic ratio $\left.C_{\infty}=2\right) .{ }^{63,70}$ We postulate the intrinsic nature of the monomer of PSU forces the chain into a more stretched conformation than for example polystyrene chains with the same molecular weight. The result is greater facility of matrix free chains to penetrate into the grafted layer.

Glass Transition Temperature. The glass transition temperature $\left(T_{g}\right)$ is considered an important property of a polymer nanocomposite. It is usually affected by the dispersion state of the nanofiller in the matrix, the specific details of surface modification or the conformation of the polymer graft on the surface nanoparticle. ${ }^{16}$ It has been reported that the addition of polymer grafted nanoparticles to polymer matrix results in either an increase or a decrease in the $T_{g}$ regardless of whether the polymers used for matrix and graft are the same or not. ${ }^{35}$ Figure 7 shows the DSC curves of PSU and nanocomposites at 5\% loading of bare and grafted $\gamma$-alu- 


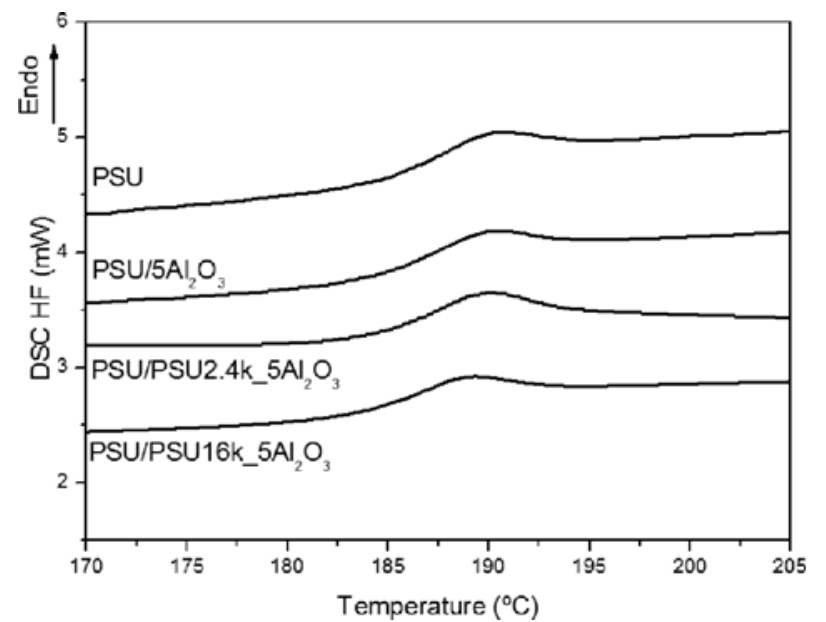

Figure 7. DSC traces corresponding to the second heating scan for the PSU, PSU $/ 5 \mathrm{Al}_{2} \mathrm{O}_{3}$, PSU/PSU2.4k_5 $5 \mathrm{Al}_{2} \mathrm{O}_{3}$ and PSU/PSU $16 \mathrm{k} \_5 \mathrm{Al}_{2} \mathrm{O}_{3}$ nanocomposites.

mina. Here, a $T_{g}$ of $185^{\circ} \mathrm{C}$ was measured for the PSU matrix and this value was unaffected by the addition of the alumina nanoparticles regardless of the modification and loading.

Neither the alumina content nor the size of aggregates or the lengths of grafts appear to cause a change in the values of the $T_{g}$, but the absence of measurable effects of nanoparticles on $T_{g}$ in densely entangled matrices like PSU is not uncommon. ${ }^{71}$ We think that the segmental mobility of PSU matrix must be so hindered that the disruption in entanglement density by the presence of the interface (named "confinement effect") seems to have no effect on the bulk polymer relaxation. A similar behavior was found here.

\section{Conclusions}

With the aim of improving the dispersibility of alumina nanoparticles within PSU matrix, PSU chains have been grafted onto alumina surface via 1,3-dipolar cycloaddition reaction between functionalized alumina with vinyl groups and terminal azide PSU chains of two different molecular weights. The grafting was completed successfully yielding moderate graft densities, 0.069 and 0.015 chains $\mathrm{nm}^{-2}$ for PSU2.4k_ $\mathrm{Al}_{2} \mathrm{O}_{3}$ and PSU16k_ $\mathrm{Al}_{2} \mathrm{O}_{3}$, respectively. Dynamic light scattering measurements of the dispersions in a good solvent (NMP), reveal that the hydrodynamic height is longer for the sample with higher molecular weight (PSU16k). Assuming a random coil conformation (mushroom regime) of the polymer grafts with a height $h \cong 2 R_{g}$, the distance between grafting points, $D_{g s}$, is smaller than the height of the grafts and only partial screening should be expected allowing nanoparticles to form aggregates.

SEM micrographs show that PSU grafted alumina particles are uniformly dispersed and perfectly embedded, however, unmodified alumina shows a poor dispersion and a bad com- patibility.

Morphologies from CLSM and FE-SEM reveal similar state of dispersion for the two types of nanoparticles, with a clear improvement in the particle size compared to bare alumina. Improvement for particles grafted with short chains, and higher graft density, is associated to the screening of core-core attraction. For the particles grafted with the longer chains, and lower graft density, the improvement may be associated to matrix chains wetting. These results suggest that the larger PSU chains have been able to entangle with the matrix during the melt processing and, consequently, their mechanical behavior should be also improved. This effect will be discussed in a future paper.

\section{$\underline{\text { Acknowledgments }}$}

Authors wish to acknowledge fund-ing from Spanish Ministerio de Economía y Competitividad under grant MAT2014-57557-R. Authors also thank to I. García and A. Cervera from Euroortodoncia for their invaluable help in nanocomposite processing.

\section{Supporting Information}

XRD diffraction pattern of surface bare alumina is provided as supplementary material. Sup-plementary information also contains FTIR spectrum for the low molecular weight PSU synthesized in this work. FTIR and TGA curves for bare alumina and VTMS modified alumina nanoparticles besides FTIR for PSU grafted nanoparticles are also presented in this section.

\section{References}

(1) D. I. Cha, K. W. Kim, G. H. Chu, H. Y. Kim, K. H. Lee, and N. Bhattarai, Macromol. Res., 14, 331 (2006).

(2) B. Gao, W. Zhang, Q. Lei, and J. Men, Macromol. Res., 21, 599 (2013).

(3) J. Wang, H. Sun, X. Gao, and C. Gao, Appl. Surf. Sci., 317, 210 (2014).

(4) M. Saxena, S. Sharma, and A. Bhattacharya, Int. J. Membr. Sci. Technol., 2, 39 (2015).

(5) S. Anaya, B. Serrano, and B. Herrero, ACS Appl. Mater: Interfaces, 6, 14460 (2014).

(6) A. J. Jose, M. Alagar, and S. P. Thomas, Mater. Manuf. Processes, 27, 247 (2012).

(7) H. Bai, Y. Zhou, and Z. Liping, Adv. Polym. Technol., 34, (2015).

(8) M. Farrokhnia, M. Rashidzadeh, A. Safekordi, and G. Khanbabaei, Iran. Polym. J., 24, 171 (2015).

(9) P. Anadão, L. F. Sato, H. Wiebeck, and F. R. Valenzuela-Díaz, Appl. Clay Sci., 48, 127 (2010).

(10) D. Olmos, S. G. Prolongo, and J. González-Benito, Compos. Part B: Eng., 61, 307 (2014).

(11) A. Dasari, Z. Z. Yu, and Y. W. Mai, Mater: Sci. Eng. R, Reports, 63, 31 (2009).

(12) L. Nayak, D. Khastgir, and T. K. Chaki, J. Mater. Sci., 48, 1492 (2013).

(13) T. S. Volkova and E. Y. Beider, Theor. Found. Chem. Eng., 
45, 717 (2011).

(14) G. Huang, J. Yang, X. Wang, and J. Gao, Macromol. Res., 21, 27 (2013).

(15) N. J. Fernandes, H. Koerner, E. P. Giannelis, and R. A. Vaia, MRS Commun., 3, 13 (2013).

(16) J. Jancar, J. F. Douglas, F. W. Starr, S. K. Kumar, P. Cassagnau, A. J. Lesser, S. S. Sternstein, and M. J. Buehler, Poly-mer (Guildf)., 51, 3321 (2010).

(17) H. R. Hakimelahi, L. Hu, B. B. Rupp, and M. R. Coleman, Polymer (Guildf)., 51, 2494 (2010).

(18) B. Zornoza, S. Irusta, C. Téllez, and J. Coronas, Langmuir, 25, 5903 (2009).

(19) Y. Devrim, S. Erkan, N. Baç, and I. Eroğlu, Int. J. Hydrogen Energy, 34, 3467 (2009).

(20) S. Kango, S. Kalia, A. Celli, J. Njuguna, Y. Habibi, and R. Kumar, Prog. Polym. Sci., 38, 1232 (2013).

(21) M. A. Hood, M. Mari, and R. Muñoz-Espí, Materials (Basel)., 7, 4057 (2014).

(22) S. K. Kumar, N. Jouault, B. Benicewicz, and T. Neely, Macromolecules, 46, 3199 (2013).

(23) J. Choi, C. M. Hui, M. Schmitt, J. Pietrasik, S. Margel, K. Matyjazsewski, and M. R. Bockstaller, Langmuir, 29, 6452 (2013).

(24) P. Akcora, S. K. Kumar, V. García Sakai, Y. Li, B. C. Benicewicz, and L. S. Schadler, Macromolecules, 43, 8275 (2010).

(25) I. Y. Jeon and J. B. Baek, Materials (Basel)., 3, 3654 (2010).

(26) S. Li, M. M. Lin, M. S. Toprak, D. K. Kim, and M. Muhammed, Nano Rev., 1, 1 (2010).

(27) R. Francis, N. Joy, E. P. Aparna, and R. Vijayan, Polym. Rev., 54, 268 (2014).

(28) A. Rungta, B. Natarajan, T. Neely, D. Dukes, L. S. Schadler, and B. C. Benicewicz, Macromolecules, 45, 9303 (2012).

(29) S. Gupta, P. C. Ramamurthy, and G. Madras, Ind. Eng. Chem. Res., 50, 6585 (2011).

(30) G. G. Vogiatzis and D. N. Theodorou, Macromolecules, 46, 4670 (2013).

(31) D. Sunday, J. Ilavsky, and D. L. Green, Macromolecules, 45, 4007 (2012).

(32) Y. Li, P. Tao, A. Viswanath, B. C. Benicewicz, and L. S. Schadler, Langmuir, 29, 1211 (2013).

(33) R. M. Bielecki, P. Doll, and N. D. Spencer, Tribol. Lett., 49, 273 (2013).

(34) K. S. Iyer and I. Luzinov, Macromolecules, 37, 9538 (2004).

(35) B. Natarajan, T. Neely, A. Rungta, B. C. Benicewicz, and L. S. Schadler, Macromolecules, 46, 4909 (2013).

(36) D. L. Green and J. Mewis, Langmuir, 22, 9546 (2006).

(37) Y. Qiao, X. Yin, L. Wang, M. S. Islam, B. C. Benicewicz, H. J. Ploehn, and C. Tang, Macromolecules, 48, 8998 (2015).

(38) N. Ogihara, K. Aoki, M. Shimizu, N. Narita, M. Okamoto, H. Kato, and S. Taruta, Nanomedicine, 7, 981 (2012).

(39) L. T. Truong, Á. Larsen, B. Holme, F. K. Hansen, and J. Roots, Polymer (Guildf)., 52, 1116 (2011).

(40) C. Dizman, S. Ates, L. Torun, and Y. Yagci, Beilstein J. Org. Chem., 6, 1 (2010).

(41) K. V. Gothelf and K. A. Jørgensen, Chem. Rev., 98, 863 (1998).

(42) H. Li, Y. Yan, B. Liu, W. Chen, and S. Chen, Powder Tech- nol., 178, 203 (2007).

(43) S. A. Hosseini, Open J. Phys. Chem., 01, 23 (2011).

(44) A. Kuznetsova, E. A. Wovchko, E. Wovchko, and J. T. Yates, Advances, 13, 5322 (1997).

(45) Y. S. Li, P. B. Wright, R. Puritt, T. Tran, Spectrochim. Acta Part A: Mol. Biomol. Spectrosc., 60, 2759 (2004).

(46) S. Oprea, High Perform. Polym., 24, 389 (2012).

(47) J. Amici, M. U. Kahveci, P. Allia, P. Tiberto, Y. Yagci, and M. Sangermano, J. Mater. Sci., 47, 412 (2012).

(48) R. Huisgen, Angew. Chem. Int. Ed. Engl., 2, 565 (1963).

(49) S. Bräse, C. Gil, K. Knepper, and V. Zimmermann, Angew. Chem. Int. Ed., 44, 5188 (2005).

(50) P. Scheiner, Tetrahedron, 24, 349 (1967).

(51) G. Socrates, Infrared and Raman Characteristic Group Frequencies, 3rd ed., John Willey and Sons, Chichester, 2001.

(52) B. J. Ash, R. W. Siegel, and L. S. Schadler, J. Polym. Sci., Part B: Polym. Phys., 42, 4371 (2004).

(53) M. Castellano, E. Marsano, A. Turturro, L. Conzatti, and G. Busca, Adsorption, 18, 307 (2012).

(54) M. Abboud, M. Fontanille, E. Duguet, M. Fontanille, J. Mater: Chem., 7, 1527 (1997).

(55) S. Yamamoto, M. Ejaz, Y. Tsujii, M. Matsumoto, and T. Fukuda, Macromolecules, 33, 5602 (2000).

(56) D. Dukes, Y. Li, S. Lewis, B. Benicewicz, L. Schadler, and S. K. Kumar, Macromolecules, 43, 1564 (2010).

(57) J. F. Moll, P. Akcora, A. Rungta, S. Gong, R. H. Colby, B. C. Benicewicz, and S. K. Kumar, Macromolecules, 44, 7473 (2011).

(58) M. J. Hore, J. Ford, K. Ohno, R. J. Composto, and B. Hammouda, Macromolecules, 46, 9341 (2013).

(59) P. Tao, A. Viswanath, Y. Li, R. W. Siegel, B. C. Benicewicz, and L. S. Schadler, Polymer (United Kingdom), 54, 1639 (2013).

(60) S. Kim, R. Mangal, and L. Archer, Macromolecules, 48, 6280 (2015).

(61) I. Borukhov and L. Leibler, Macromolecules, 35, 5171 (2002).

(62) Pierre-Gilles Gennes, Scaling Concepts in Polymer Physics, Cornell University Press, Ithaca, NY, 1979.

(63) Silvia Ioan, Functionalized Polysulfones: Synthesis, Characterization, and Applications; CRC Press, Boca Raton, Florida, 2015.

(64) V. G. V. Schulz and I. Einleitung, Physics (College. Park. Md)., 29, 93 (1958).

(65) M. N. Tchoul, S. P. Fillery, H. Koerner, L. F. Drummy, F. T. Oyerokun, P. A. Mirau, M. F. Durstock, and R. A. Vaia, Chem. Mater, 22, 1749 (2010).

(66) G. Henn, D. G. Bucknall, M. Stamm, P. Vanhoorne, and R. Je, Macromolecules, 29, 4305 (1996).

(67) R. Hasegawa, Y. Aoki, and M. Doi, Macromolecules, 29, 6656 (1996).

(68) P. Auroy, L. Auvray, and L. Léger, Phys. Rev., 66, 2769 (1991).

(69) T. B. Martin, P. M. Dodd, and A. Jayaraman, Phys. Rev. Lett., 110, 1 (2013).

(70) J. Roovers, R. Ethier, and P. M. Toporowski, High Perform. Polym., 3, 151 (1990).

(71) M. Wang, C. Y. Yue, and B. Chua, J. Mater. Sci. Mater. Med., 12, 821 (2001). 